\title{
IoT based Smart Laboratory System
}

\author{
Amruta N. Banagar \\ Department of Computer Engineering, \\ Fr. C. Rodrigues Institute of Technology, \\ Vashi, India
}

\author{
Rajshankar Khattar \\ Department of Computer Engineering, \\ Fr. C. Rodrigues Institute of Technology, \\ Vashi, India
}

\begin{abstract}
Internet of Things (IoT), an embedded technology helps enable breakthrough services by interconnection of physical and virtual things, intelligent interaction and Machineto-Machine (M2M) communication between environments, objects and interoperable information technologies. IoT has found many applications in the fields of medicine, transportation, farming, manufacturing and automation. In the present scenario, the appliances of a laboratory like lights and fans are left on, even when not in use, which leads to a rise in power consumption of the laboratory. IoT can be used to automate the laboratory which will help in effective power consumption, minimal human assistance required and easy monitoring of the laboratory. This paper proposes a Smart Automation System for the department's computer laboratory. The system will monitor the changes like temperature and lighting conditions of the environment, produce analyzed results by controlling the appliances as well as report abrupt changes.
\end{abstract}

Keywords-Automation; Embedded Technology; IoT; Machine-to-Machine (M2M) Communication; Smart Laboratory;

\section{INTRODUCTION}

The term Internet of Things (IoT) was first coined by Kevin Ashton in the year 1999 in the background of supply chain management. IoT represents the ability of network devices to sense and gather knowledge from the environment, this knowledge is then shared across the Internet where it can be processed and utilized for an extensive range of applications like healthcare, utilities, home automation, transportation, defense, public safety, wearables and augmented reality (AR). It is expected that IoT devices will rise to 26 billion by the year 2020, which is 30 times the estimated number of devices deployed in the year 2009. With the help of IoT, automation of processes is possible. The smart sensor networks which are connected to a cloud or local system can provide us with better control of the processes.

In the current scenario, there is a lot of unnecessary wastage of electricity in the laboratory. The appliances of the laboratory are left switched on even when not required. This leads to a rise in electricity consumption and hence exorbitant electricity bills.

Our objective is to build a Smart Laboratory which takes into consideration environmental parameters such as temperature and light intensity of the laboratory. With the help of these parameters, the system will analyze and process the information collected and then produce appropriate results. This will help in automation and effective power consumption of the laboratory. The system will be able to adapt to the environment and act accordingly. This means the usage of the appliances will be done only when required. The automation of the laboratory will help in the efficient utilization of the appliances with minimal human assistance.

\section{THEORY}

\section{A. Study of IoT}

The IERC [1] (European Research Cluster on the Internet of Things) definition is as follows - "IoT is a dynamic global network framework with self-configuring capabilities based on standard and interoperable communication protocols where physical and virtual 'things' have identities, physical attributes, and virtual personalities and use intelligent interfaces, and are seamlessly integrated into the information network."

IoT is mainly an environment of a diversity of things having a wide presence, which through wireless and wired connections are able to interact with each other and cooperate with other things to create new applications and reach common designated goals.

\section{B. Architecture of IoT}

The architecture of Internet of Things [2] is designed to stem a wide spectrum of data at any given point of time. It is an eminently decisive framework that can connect with any aspect of the smart device.

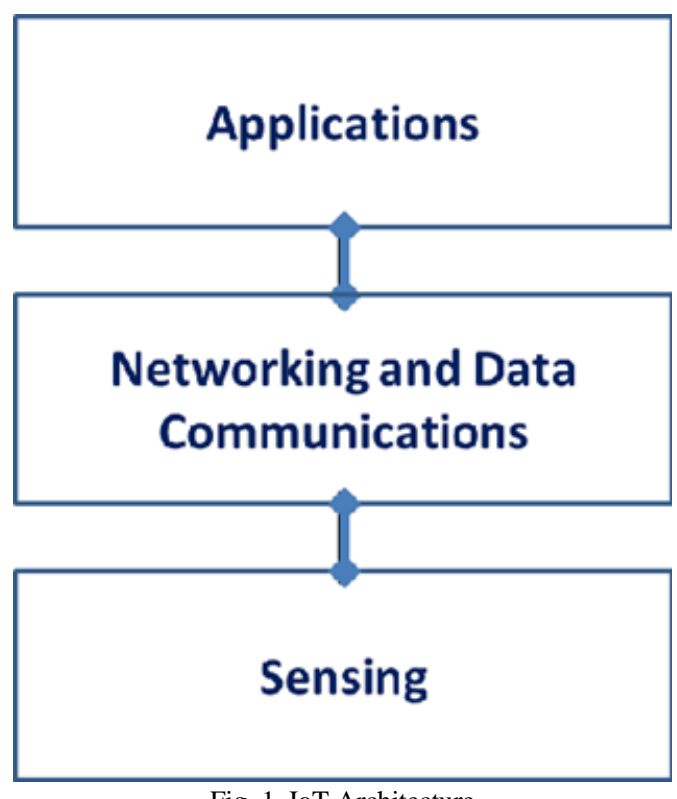

Fig. 1. IoT Architecture

The base layer is the sensing and identification layer. Every device which senses the environment and uniquely identifies the sensed data belongs to this layer. The sensing level focuses 
on gathering information via the sensors. The sensors are small in size, inexpensive and highly available because of which, they can be embedded into mobile computation devices, wearables and autonomous machines. The environmental factors such as humidity, temperature, light, pressure and vibration can be captured with the help of the sensors.

The next layer is the network construction layer. This layer is responsible for operational handling of the IoT data. Routing of the information is done with the help of this layer. This can be anything like wireless LAN, wireless MAN, wireless wide-area network, wireless personal area network, Internet, etc. The sensed data is accumulated and then sent to the next layer by the network layer. By aggregating the data at the gateway, summarizing and analyzing, the network layer can curtail the volume of irrelevant data to be forwarded on.

The topmost layer is the Application layer - responsible for illustrate and interpret the data. It reacts to its interpretation accordingly. We can think of an example wherein, if the temperature sensor in a smart home senses the temperature of the room to be increasing, then the air conditioner will be switched on promptly.

\section{Applications of IoT}

The applications of IoT [1] are as follows:

1. Wearables

- Smart bands

- Wireless ECG monitor

- Smart contact lens

- Smart devices helping people to quit smoking

2. Smart Healthcare

- With the help of IoT, improved care for patients and providers can be facilitated.

3. Smart Clothing

- Smart fabrics can communicate with the smartphones to process biometric information such as heart rate, body temperature, breathing patterns, stress levels, hormone levels and the movement of body, with real time feedback.

4. Smart Buildings

- IoT in architecture of buildings can help in accelerating development, adding intelligence across building operations, lower operational costs and consolidating workloads to build smarter offices, industries, residents, hotels, hospitals, etc.

\section{Smart Energy}

- Economical electricity transmission

- Immediate electricity restoration after power disruptions

- Decreased operational and management costs

- Controlled demand

6. Smart Mobility and Transport

- The transport with the help of internet connected vehicles is effortless, hassle-free and safer. IoT provides cuttingedge services relating to different modes of transport and enables user to be informed about the environment and make safer and smart decisions and thereby make optimum use of the transportation networks.
7. Smart Manufacturing

- Digitized manufacturing systems.

8. Smart Farming

- Viable farming

- Increased production with much lesser costs

- Lesser environmental impact

\section{Advantages of IoT}

- Communication

- Communication between physical devices is possible with the help of IoT and this leads to transparent systems which have better productivity and greater quality.

- Automation and Control

- Without the intervention of humans, faster and timely output is achieved by automating machines which control vast amounts of information.

- Monitoring

- Smart sensors are used in monitoring different aspects of our daily life for assorted applications which successively saves time and money.

- Better aspect of living

- Applications based on IoT are able to increase convenience, comfort and better administration in our daily life.

- New business opportunities

- New business opportunities are created with the help of IoT technology; hence this increases monetary growth and new opportunities for jobs.

- Better environment

- IoT helps in saving natural resources, trees and in creating a greener and sustainable planet.

\section{PROPOSED METHODOLOGY}

\section{A. Working of Existing System}

A few systems have been proposed to apply IoT technology on laboratories.

Reference [3] shows a Smart Laboratory implementation which is built across IoT and Mobile communication technologies to supervise the overall activities of the laboratory including power consumption and application of devices, sensing environmental parameters, thereby providing a smart environment with balanced energy consumption and comfort. The system monitors the consumption pattern of the devices and uploads it to their server and establishes remote control of appliances from anywhere thereby reducing power wastage.

Reference [4] shows a smart laboratory implementation for management of subsystems of a university, including lighting conditions, air conditioning, heating, audio/video, controlling switches and security. It highlights the benefits for university and the students using the smart laboratory.

Reference [5] shows a Secure and Smart Laboratory implementation with Wireless Sensor Network (WSN). The system is implemented using an Ambient lighting module. It uses Passive Infrared Sensor (PIR) and Environment Sensor (ES) for security. The difficulty faced by the system is that, WSN has limited computation, lack of communication 
resources, reduced power, shortage of storage, prone to attacks and lower bandwidth to communicate.

\section{B. Block diagram of Smart Laboratory system}

Fig. 2. shows the block diagram of the Smart Laboratory system. The data from the environment will be sensed with the help of a motion sensor, luminosity sensor, temperature and humidity sensor. The data from the sensors will then be transmitted to the microcontroller - Raspberry Pi version 3B. The microcontroller processes the data.

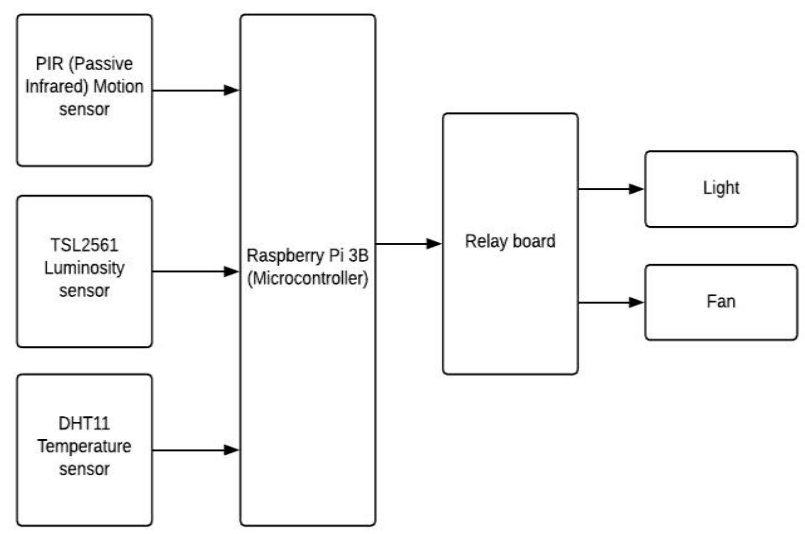

Fig. 2. Block diagram of the system

Based on the processing, switching of tube lights and fans will be done accordingly with the help of the relay board.

\section{Flowchart of Smart Laboratory system}

Fig. 3. depicts the flowchart of the Smart Laboratory system which shows the working of the system according to the laboratory timings.

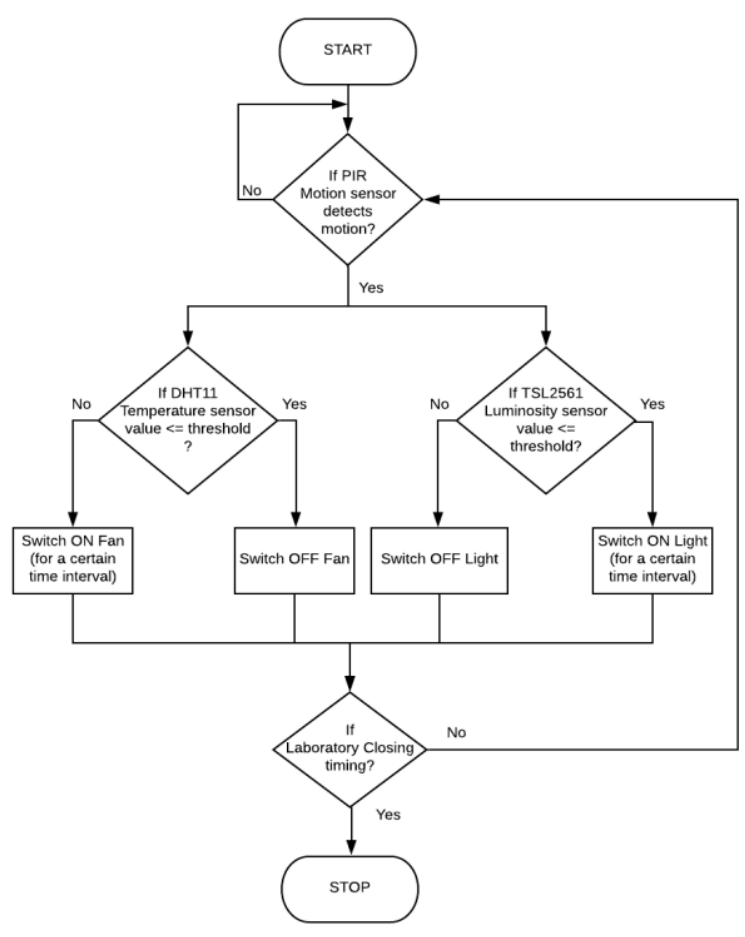

Fig. 3. Flowchart of the system

\section{Smart Laboratory Implementation using Raspberry Pi}

The Smart Laboratory System will sense the motion in the environment using the PIR sensor. When the motion is detected, the temperature and the light intensity from the environment is sensed using the DHT11 temperature sensor and TSL2561 luminosity sensor. If the value of the temperature is beyond a certain threshold, then the fans of the laboratory will be switched on for a certain time interval, else switched off. Similarly, if the luminosity value is beyond a certain threshold, then the tube lights in the laboratory will be switched off for certain time interval, else switched on.

When no motion is detected, then tube lights and fans of the laboratory will be switched off until motion is detected again.

The data from the sensors will be sent to the microcontroller Raspberry Pi version $3 \mathrm{~B}$. The processing will be done by the microcontroller and appropriate actions will be taken (controlling tube lights and fans).

The data from the microcontroller will be sent to the cloud platform for constant monitoring and analyzing purposes. Based on the usage, patterns will be detected and the threshold values of temperature and light intensity will be modified accordingly.

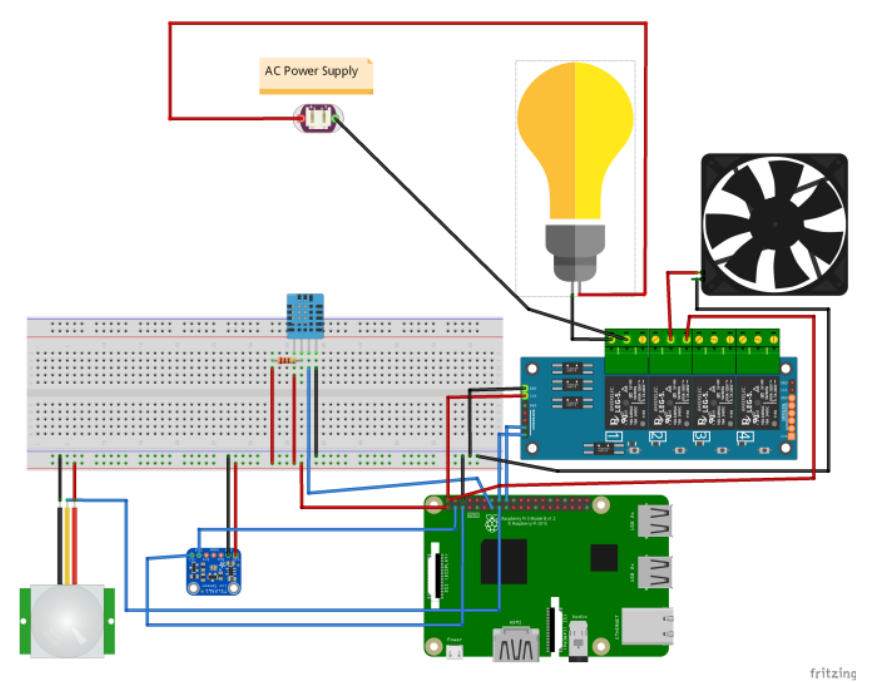

Fig. 4. Circuit Diagram of the System

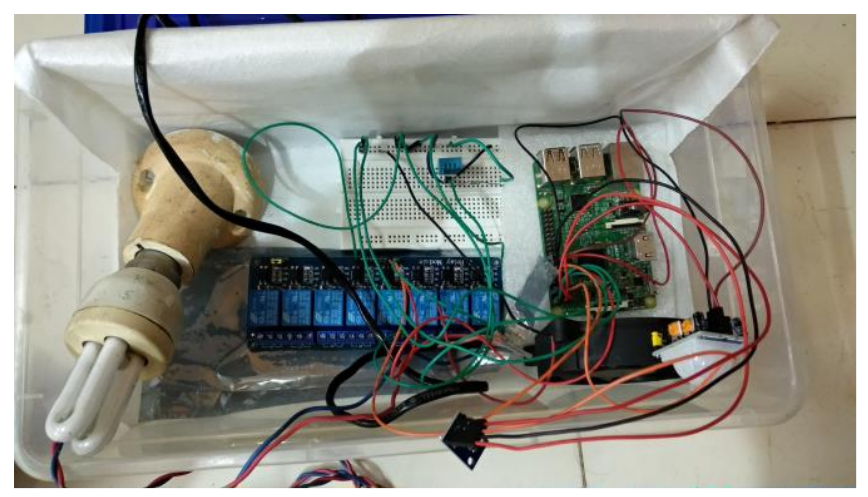

Fig. 5(a). Model Components 
Fig. 4. depicts the circuit connections of the Smart Laboratory Model. The components of the model comprise of:

1. Raspberry Pi 3B

2. 8 channel Relay Board

3. TSL 2561 - Luminosity sensor

4. DHT11 - Temperature \& Humidity sensor

5. Passive Infrared (PIR) Motion Sensor

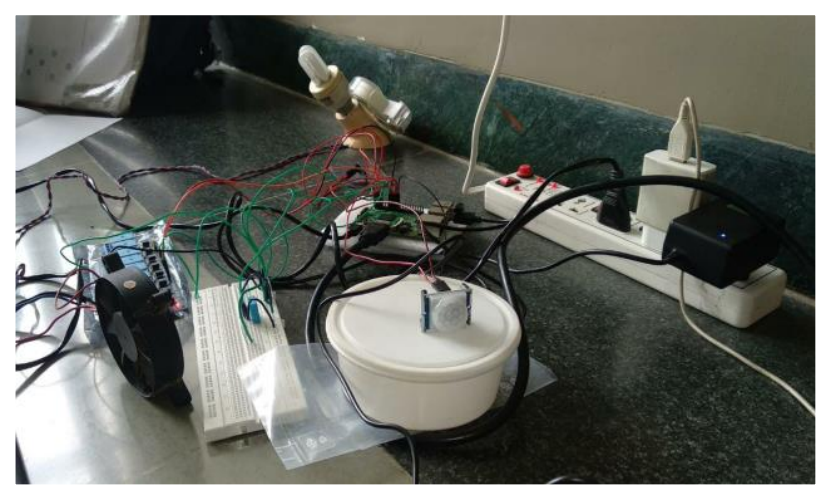

Fig. 5(b). Model of the System

Fig. 5(a) and (b) represent the model for the Smart Laboratory system consisting of all the components as stated above.

\section{RESULT ANALYSIS}

It was observed from the Smart Laboratory system's working that when the lights and fans were not required, they were switched off by the system automatically. This helped in reducing power wastage and misuse unlike earlier in the past. Due to automation of the laboratory, human assistance was not required to switch off appliances when they were not in use. The IoT based Smart Laboratory led to the smooth functioning and efficient usage of the electronic appliances of the laboratory.

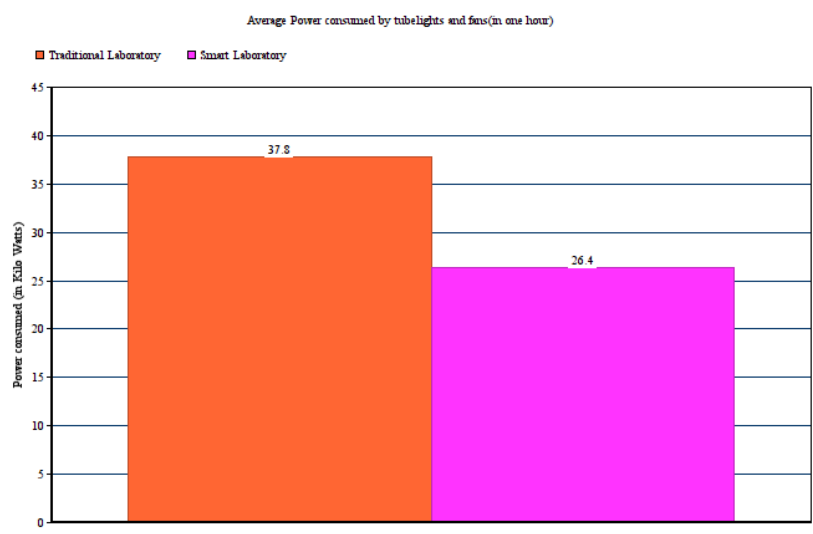

From Fig. 6., it can be observed that the average power consumption in an hour is reduced approximately by $30 \%$ for the smart laboratory over the traditional laboratory.

\section{CONCLUSION AND FUTURE SCOPE}

The system extends the home automation technology to the college laboratories, and hence to create a smart laboratory. The smart laboratory reduces the amount of power consumed throughout the day. Thus, the system helps in managing energy efficiently. Also, the automation of the laboratory leads to its improved organization and reduces staff involvement in simple administration tasks.

Challenges faced are as follows:

- Complexity

- IoT being a varied and complicated network, any breakdown or bugs in the hardware or software can lead to serious issues. Even a power failure can lead to a lot of hindrance.

- Privacy and Security

- Invasion of IoT devices can be done in many ways, which involve apps that first obtain access to IoT application, then start to monitor, control, and tamper the device. IoT often comes with unprecedented issues such as brute forcing, issue of default passwords, system invasions, remote access and untrustworthy communication.

The future scope considers the security concerns of the IoT system and tries to implement security and privacy of users' data using the Blockchain technology on top of IoT system.

\section{REFERENCES}

[1] Patrick Guillemin, Martin Serrano, Mustapha Bouraoui, Luis P'erez Freire, Thomas Kallstenius, Kit Lam, Markus Eisenhauer, Klaus Moessner, Maurizio Spirito, Elias Z. Tragos, Harald Sundmaeker, Pedro Malo, Ovidiu Vermesan, Peter Friess and Arthur van der Wees. IoT Digital Value Chain Connecting Research, Innovation and Deployment, IERC Cluster SRIA 2016, online at http://www.internet-of-thingsresearch.eu/, pp. 20-80.

[2] IEEE Internet initiative, https://iot.ieee.org/, Towards a definition of the Internet of Things (IoT), Revision 1 - published May 2015, pp. 10-16.

[3] A. Rajeswari, M. Poongothai, P. Muthu Subramanian. Design and Implementation of IoT Based Smart Laboratory, IEEE, 2018.

[4] Hu Yin. Smart Lab Technologies, Springer, Berlin, Heidelberg, 2015.

[5] Hitesh Kumar, P Mary Cherian. Implementation of a Secure and Smart Lab with Wireless Sensor Network, IJSR, 2014.

Fig. 6. Average Power consumption (in 1 hour) 